\title{
A Novel Treatment of Symptomatic Menopause Autologous Ovarian Transplantation Research in Progress
}

\author{
Boris Petrikovsky*1, EV Zharov ${ }^{2}$ and A Ansari ${ }^{3}$ \\ ${ }^{1}$ Professor and former Chairman of Obstetrics and Gynecology, Nassau University Medical Center, USA \\ ${ }^{2}$ Hospital of Russian Federation Academy of Science, USA \\ ${ }^{3}$ Former Director of Gynecology, Nassau University Medical Center, USA
}

*Corresponding author: Boris Petrikovsky, Professor and former Chairman of Obstetrics and Gynecology, Nassau University Medical Center, USA.

To Cite This Article: Boris Petrikovsky. A Novel Treatment of Symptomatic Menopause Autologous Ovarian TransplantationResearch in Progress. Am J Biomed Sci \& Res. 2019 - 3(3). AJBSR.MS.ID.000679. DOI: 10.34297/AJBSR.2019.03.000679

Received: April 04, 2019 | Published: June 13, 2019

\section{Introduction}

Following the onset of menopause, some women suffer from significant effects, including hot flashes, osteoporosis, mood swings, loss of libido, among others. While natural menopause occurs at the age of 50 plus, on occasion this process may be induced at age 40 or less due to premature menopause [1,2].

\section{Materials and Methods}

After obtaining informed consent, partial opportunistic oophorectomy was performed laparoscopically or by laparotomy as a part of indicated cesarean section or gynecological operation. After its removal, the ovary was placed in a biohazard bag containing cold, sterile Hanks balanced salt solution. Working in a fume hood and using standard sterile techniques, the ovarian cortex was dissected from the stroma and cut into $1 \times 0.5 \mathrm{~cm}$ strips. Each strip was placed in a cryovial. The vial was filled with protective solution and equilibrated on a rotating mixer at 4 Q $\mathrm{C}$ for 30 minutes. These vials were then placed into a -80 a $\mathrm{C}$ freezer in a styrofoam covered box where the temperature dropped gradually over a 24-hour period. At the end of this period, the specimens were transferred to a cryofreezer cane and placed inside cryoflex tubing. Separate pieces of tissue were sent at times to pathology for light and electron microscopy to ascertain their condition.

\section{Results}

In 2000, we established an ovarian tissue cryopreservation bank in collaboration with Professor E. Zharov (Russian Federation) with the goal to preserve for future auto transplantation ovarian tissue retrieved with the patient's consent during indicated obstetrical or gynecological procedures (cesarean section-15, minilaparotomies and tubal ligation-12, gynecological surgeries for benign conditions-22). None of the patients experienced complications directly related to opportunistic ovarian sampling. Now, 20 years later, histological assessment of preserved ovarian strips (15 samples) demonstrated ovarian tissue adequate for reimplantation, 6 samples contained visible icicles and were judged unfit for transplantation.

At a later time (15 years later) or when medically indicated, strips of cortical tissue were thawed, prepared by washing in a $1 \%$ human albumin mixed with normal saline sterile solution to be inserted into a subcutaneous pocket of a postmenopausal symptomatic patient. Fifteen-year-old specimens were reassessed and in $85 \%$ of cases were found to be suitable for auto transplantation.

\section{Discussion}

In 2000, we initiated an IRB approved research protocol at Nassau University Medical Center Petrikovsky BM, Ansari AH, Beers PG, et al. which stated as follows: "After obtaining approval from the Institutional Review Board, patients, ages 40 and under, were included in this study. Normal ovarian function in these cases was established prior to ovarian sampling, using such methods as; hormonal assay, pelvic ultra-sonography and endometrial sampling if indicated clinically. To the best of our knowledge, this report is the first one to provide the utility of long-term (15-20 years) successful freezing of ovarian tissue. We believe it may be used to treat menopausal symptoms in carefully selected and motivated patients. The long-term outcomes of ovarian auto transplantation to treat and/or prevent menopause symptoms, remain unclear and require further research.

\section{References}

1. Oktay K, Gill AP, Zhang J (2010) Who is the best candidate for oocyte cryopreservation research? Fertil Steril 93(1): 13-15. 
2. Petrikovsky BM, Zharov EV, Cohen M (2019) Individualized hormone replacement therapy versus ovarian transplantation. World J Gynecol \&
Women's Health 1(5): 1-2. 